\title{
Fibrin-based Biological Adhesives Prevent Anastomotic Leakage in Rectal Anastomoses: Prospective Multicentre Randomised Clinical Trial
}

\author{
Jesús Lago Oliver ${ }^{1,}$, , Salvador Argudo Garijo ${ }^{2}$, Mauricio Burneo Esteves ${ }^{1}$, Irene Arjona Medina ${ }^{3}$, \\ Mercedes Sanz Sánchez ${ }^{1}$, Jorge Martín Gil ${ }^{1}$, Fernando Turégano Fuentes ${ }^{4}$, Antonio Torres García ${ }^{5}$ \\ ${ }^{1}$ Department of Surgery, University General Hospital Gregorio Marañón, Madrid, Spain \\ ${ }^{2}$ Department of Surgery, Southeast Hospital, Madrid, Spain \\ ${ }^{3}$ Department of Surgery, General Hospital of Ciudad Real, Ciudad Real, Spain \\ ${ }^{4}$ Department of General Surgery II, University General Hospital Gregorio Marañón, Madrid, Spain \\ ${ }^{5}$ Department of Surgery, San Carlos University Hospital, Madrid, Spain
}

Email address:

jesus.lago@salud.madrid.org (J. L. Oliver)

To cite this article:

Jesús Lago Oliver, Vador Argudo Garijo, Mauricio Burneo Esteves, Irene Arjona Medina, Mercedes Sanz Sánchez, Jorge Martín Gil, Fernando Turégano Fuentes, Antonio Torres Garcí. Fibrin-based Biological Adhesives Prevent Anastomotic Leakage in Rectal Anastomoses:

Prospective Multicentre Randomised Clinical Trial. Journal of Surgery. Vol. 3, No. 3, 2015, pp. 26-31. doi: 10.11648/j.js.20150303.13

\begin{abstract}
Background: Fibrin-based biological adhesives are used for tissue adhesion improving the outcome of gastrointestinal sutures. The objective was to assess the effectiveness of fibrin-based biological adhesives for prevention of anastomotic leakage in high-risk gastrointestinal anastomoses. Methods: A randomized clinical trial was designed to recruit patients underwent a rectal resection surgery. A subgroup of patients with rectal anastomosis were recruited from 2 different hospital centres. Patients in which a biological fibrin-based biological adhesive was applied to the suture line (study group) were compared versus a control group under standard practice. The main outcome measures was presence or absence of leakage and need for reoperation. Results: Thirty seven patients underwent a rectal resection and anastomosis. In 21 standard practice was applied and a fibrin-based adhesive was used in 16. Fourteen patients (37.8\%) had a clinical or subclinical anastomotic leak, 11 belonging to control group versus 3 patients in the study group ( $p$-value of 0.04 ). Statistically significant difference ( $p$-value of 0.048 ) in the need for reoperation. We found no association between the use of drains and anastomotic leakage. There were only 3 leaks in the group in which a drain was placed, as compared to 11 leaks in the group in which no drain was placed, but these findings were probably due to chance $(p=0.54)$. Conclusions: The use of these adhesives could not only reduce serious postoperative complications related to dehiscence but also improve the prognosis and oncological outcome of rectal and sigmoid cancer treatment.
\end{abstract}

Keywords: Fibrin Tissue Adhesive, Anastomotic Leak, Digestive System Surgical Procedures, Randomized Controlled Trial, Spain

\section{Introduction}

One of the greatest challenges that colorectal surgeons face in their daily clinical practice is the high rate of rectal anastomosis leakage with its devastating effects. A great number of procedures have been described to try to decrease the leakage rate and the mortality and morbidity associated with this serious complication [1-4]. Several published research works describe the well-known influence of the surgeon's experience on the outcomes of rectal surgery [5], while other publications stress the need to perform a protective ileostomy in certain cases [6]. Furthermore, others report the association of anastomotic leakage with the number of endostapler cartridges used for rectal resection [7].

However, despite the great advances achieved in colorectal surgery and also in the oncological outcomes of rectal cancer treatment, we still have advanced little in improving the healing of rectal sutures that would allow for significant reductions in the leakage rate. This would not only allow for improved postoperative outcomes but also for avoiding cancer treatment delay for these patients and even reducing primary 
tumour recurrence rate as a direct result of dehiscence, as evidenced by many recent publications $[8,9]$.

We have made advances in overall patient care; however, we have focused on the mechanical reinforcement of our sutures to try to reduce the risk of poor healing. Unfortunately, we have made little progress in this direction. Increasing mechanical reinforcements with manual sutures or staplers and cutting devices and increasing the number of suture planes or staple rows have only slightly decreased the number of anastomotic dehiscence cases [10,11].

Fibrin-based biological adhesives, which are substances classified as drugs in their Summary of Product Characteristics, are used for tissue adhesion. These adhesives have been used for decades to seal different tissues in an attempt to decrease lymphorrhagia or serous effusions [12]. They have also been used, as indicated in their Summary of Product Characteristics, as sealants to stop bleeding or to prevent bile leak in liver resections [13], and they have even been used to fix the mesh in hernia repair [14,15] and to prevent bowel adhesion to the edges in laparoscopic repair [16].

Conversely, there are reports that point to a mechanism closer to drug action than to a purely mechanical one. In this sense, these adhesives might cause healing induction apart from the purely mechanical direct tissue adhesion [17,18]. Literature references on this theoretical mechanism of action have raised the possibility that fibrin-based biological adhesives might promote healing and improve the outcome of gastrointestinal sutures [19-21].

With this background, we designed a clinical trial to demonstrate the effectiveness of using fibrin-based biological adhesives in the prevention of anastomotic leakage in high-risk gastrointestinal anastomoses. The present research work evaluates patient results in a subgroup with rectal anastomosis.

\section{Materials and Methods}

\subsection{Protocol}

All patients scheduled for an elective or urgent surgery procedure, in which a high-risk anastomosis would be considered, were recruited for inclusion in the study. The following were considered high-risk gastrointestinal anastomoses: rectal anastomosis, gastrojejunal anastomosis in bariatric surgery, oesophageal anastomoses in the absence of gastrointestinal repair, and anastomoses of an obstructed intestinal segment at the time of surgery.

Patients were recruited from 3 hospitals in Madrid, as follows: University General Hospital "Gregorio Marañón", Southeast Hospital, and San Carlos University Hospital. Patients who met the inclusion criteria and did not meet any exclusion criteria were included for analysis using the "intention-to-treat" principle.

Patients with rectal anastomosis were recruited from 2 of the 3 centres included in the clinical trial, as follows: University General Hospital "Gregorio Marañón" and
Southeast Hospital. The protocol was approved by the Area1 Committee of Ethics and Clinical Research of Madrid and the Spanish Agency of Medicines with protocol code PROTISSUCOL001, registration number 25/06, and EudraCT registration number 2007-003006-98, and it has also been registered at www.clinicaltrials.gov with registration number NCT01306851. The study was performed in accordance with the ethical standards laid down in the Declaration of Helsinki.

Once the informed consent form was signed, if the patient met the inclusion criteria and did not meet any criteria for preoperative exclusion, the patient was included in the study, and randomisation was performed using a table of random numbers generated in Excel ${ }^{\circledR}$ for Windows for the entire study, assigning patients to one of two groups, as follows: standard treatment group (control group) or standard treatment group, in which a biological fibrin-based biological adhesive was applied to the suture line (study group).

\subsection{Surgical Technique}

The subgroup of patients evaluated in these preliminary results underwent a rectal resection surgery due to rectal or sigmoid colon cancer, the treatment of which required a partial resection of the rectum. Most resections included a rectal anastomosis not lower than the middle third of the rectum, which in each case was performed by a circular mechanical anastomosis of 29 or $32 \mathrm{~mm}$. In 12 cases, a protective ileostomy was performed. In all patients, the tightness of the anastomosis was confirmed with air and serum. In the study group, once the rectal mechanical anastomosis was performed, a fibrin-based biological adhesive was applied on the outside of the entire circumference $\left(5 \mathrm{ml}\right.$; Tissucol duo ${ }^{\circledR}$ Baxter headquartered in Vienna, Austria) (Figure 1). Some patients required drainage, according to the surgeon's criteria. The surgeons who took part in the study were selected based on maintaining uniform criteria and a standard homogeneous surgical technique.

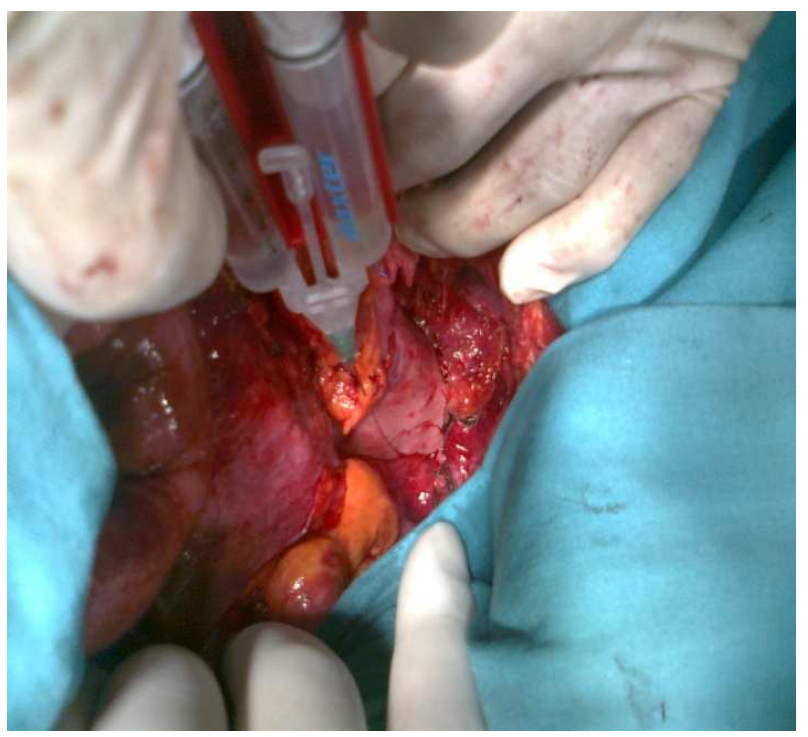

Figure 1. Tissucol application in colorectal anastomosis. 


\subsection{Postoperative Management}

All patients underwent an imaging test, in case of possible anastomosis leakage, to confirm the diagnosis, except for those patients who had to be reoperated due to a clinical diagnosis. In case of a suspected leakage, a contrast computed tomography (CT) scan was used as a diagnostic imaging test. In the remaining patients who were not suspected of leaking, a barium enema with water-soluble contrast was performed on the seventh postoperative day to diagnose all subclinical leaks, which were interpreted in all cases as anastomotic leakage, even in patients without symptoms or suspected dehiscence.

\subsection{Definitions}

We defined an anastomotic leakage or impaired healing of the suture, in general, by the following: a) the CT scan and barium enema radiology report of anastomotic dehiscence, in the context of an imaging test examined by an expert radiologist not involved in the clinical trial and not knowing the group to which the patient was assigned; b) a reoperation in which leakage is present; c) the presence of faecal material in the drainage fluid; or d) a combination of several of these findings.

\subsection{Control of Variables}

The following data were collected at the time of patient enrolment and prior to randomisation: all demographic data; pathological variables; comorbidity; preoperative risk factors; control analytical data; surgical technique; intraoperative incidents, both when performing the anastomosis and when using fibrin-based biological adhesive; use or not of drains; postoperative course; postoperative control analytical data; postoperative imaging control; the need for reoperation and its findings; and clinical evidence of progression until patient discharge. Readmissions and late leaks were also collected.

\subsection{Statistical Analysis}

Statistical analyses were performed with the PASW $^{\circledR}$ Statistics 18.0 (Predictive Analytics Software) statistical package. For all analyses, an alpha value $(\alpha)$ of 0.05 has been considered significant, i.e., the p-value of the statistical test should be equal to or less than 0.05 to consider a significant difference.

A descriptive analysis of the sociodemographic, clinical, and informational variables regarding the management of the fibrin-based biological adhesive was performed. For the categorical or qualitative variables that were considered possible risk factors for patients, percentages and frequency distributions were calculated. For quantitative variables, such as age, the mean, standard deviation, and minimum and maximum deviations were calculated. Alternatively, a comparison of means has been performed to verify that there were no previous significant differences between groups (study group and control group).

Subsequently, a comprehensive statistical analysis of patients who had anastomotic leakage was performed by analysing their demographics and whether or not a fibrin-based biological adhesive was applied to them. In bivariate analyses, the variables considered fundamental (leaks/use-no use of adhesive) were crossed. In addition, the Pearson's $\chi 2$ statistic was calculated to test the hypothesis of independence between these variables.

\subsection{Primary "Endpoint" of the Study}

The primary "endpoint" of the study was to demonstrate the presence or absence of leakage at 6 months.

\section{Results}

From March 2007 until November 2010, a total of 106 patients who met the inclusion criteria were recruited in the 3 General Surgery departments of the participating hospitals for the clinical trial. Patients who met these criteria and signed the informed consent form were randomised preoperatively to be assigned to the study group or the control group. Of these 106 patients, 2 were excluded for presenting peritoneal carcinomatosis, one of the intraoperative exclusion criteria, at the time of the intervention.

Of the 104 patients evaluated, 37 patients underwent a rectal resection and anastomosis, which was performed in the middle or upper rectum in 25 patients. All patients underwent a rectal resection for malignancy, with a primary anastomosis performed on all of them. All 12 patients who underwent a lower rectal anastomosis had a protective ileostomy performed.

The mean age was 64 years (std. deviation: 16, median: 69) with a homogeneous distribution by sex. Comorbid risk factors were the most commonly associated variable with a poor healing of the anastomosis. Fifty percent $(50 \%)$ of patients had cardiovascular history, which was most frequently arterial hypertension. Additionally, $21.6 \%$ of patients had diabetes mellitus.

Both study groups (patients who were assigned post randomisation to the study group or control group) were comparable in sociodemographic data, disease to be treated, type of intervention, and comorbidity. Both groups were comparable in terms of type of surgery, resection technique, type of anastomosis, intervention indication, surgical equipment, preoperative analytical data, and nutritional parameters.

The postoperative results that were compared between the two groups are listed in Table 1. As seen in the table, 16 patients who underwent a rectal resection belong to the study group, while 21 patients belong to the control group, this difference was no statistically significant. Operations were performed laparoscopically in 27 patients and by open surgery in 10 patients, with no significant differences in the distribution between the two groups.

Fourteen of the 37 patients $(37.8 \%)$ had a clinical or subclinical anastomotic leak that was detected in those cases by a barium enema performed on the seventh postoperative day according to the protocol. As can be seen in Table 2, the leak rate was much higher in the control group (52.4\%) 
compared to the study group $(18.7 \%)$ in which the fibrin-based biological adhesive was used, with 11 patients in the control group (no adhesive) having an anastomotic leakage versus 3 patients in the study group (adhesive). This difference is statistically significant, with a p-value of 0.04 .

Conversely, it is obvious that a p-value of 0.048 also indicates a statistically significant difference in the need for reoperation between the application or not of a fibrin-based biological adhesive. Of the 16 patients to whom a fibrin-based biological adhesive was applied, only 2 patients (12.5\%) required a reoperation. Of the 21 patients to whom the adhesive was not applied, 9 underwent a reoperation for complications related to the anastomosis $(42.9 \%)$,(Table 3$)$.

Another objective of the secondary assessment was the use of abdominal drains. The conclusions after the results were studied are limited, as this was not a controlled "endpoint" of the study, and their use was left to the surgeon's criteria. In the general data of our study, the use of drains was a protective factor for an anastomotic leak, having found significant differences. However, when analysing the subgroup of rectal anastomosis, we found no association between the use of drains and anastomotic leakage. There were only 3 leaks in the group in which a drain was placed, as compared to 11 leaks in the group in which no drain was placed, but these findings were probably due to chance $(\mathrm{p}=0.54)$ (Table 4$)$.

Finally, when analysing mortality (Table 5), we found no significant differences between both groups. Five patients died of complications related to the suture, with 1 in the study group and 4 in the control group $(p=0.27)$.

Table 1. Surgical times and postoperative stays.

\begin{tabular}{|c|c|c|c|c|c|c|c|c|c|c|c|}
\hline \multirow{3}{*}{$\begin{array}{l}\text { Post-Operative } \\
\text { Results }\end{array}$} & \multirow{2}{*}{\multicolumn{3}{|c|}{$\begin{array}{l}\text { Patients } \\
\text { Tissucol use }(n=16)\end{array}$}} & \multicolumn{3}{|c|}{ Patients } & \multirow{2}{*}{\multicolumn{3}{|c|}{ Total }} & \multirow{3}{*}{$\begin{array}{l}\text { Parametric } \\
\text { P-value }\end{array}$} & \multirow{3}{*}{$\begin{array}{l}\text { Non- } \\
\text { parametric } \\
\text { P-value }\end{array}$} \\
\hline & & & & \multicolumn{3}{|c|}{ No Tissucol use $(n=21)$} & & & & & \\
\hline & Mean & $95 \% \mathrm{C}$ & & Mean & $95 \% \mathrm{C}$ & & Mean & $95 \% \mathrm{C}$ & & & \\
\hline Surgical time & 259.06 & 217.54 & 300.58 & 233.10 & 195.12 & 271.07 & 244.32 & 217.31 & 271.34 & 0.34 & 0.43 \\
\hline Days in the ICU ${ }^{\mathrm{a}}$ & 0.13 & 0 & 0.39 & 0.10 & 0 & 0.23 & 0.11 & 0 & 0.24 & 0.84 & 0.77 \\
\hline $\begin{array}{l}\text { Total days of } \\
\text { post-operative stay }\end{array}$ & 10.41 & 7.36 & 13.44 & 7.14 & 4.54 & 9.74 & 8.55 & 6.60 & 10.51 & 0.09 & 0.03 \\
\hline
\end{tabular}

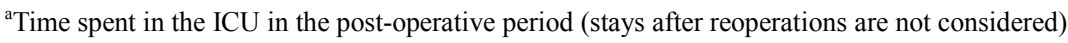

Table 2. Leaks.

\begin{tabular}{lllll}
\hline \multirow{2}{*}{ Leaks } & $\begin{array}{l}\text { Tissucol use } \\
(\mathbf{n = 1 6 )}\end{array}$ & $\begin{array}{l}\text { No Tissucol use } \\
(\mathbf{n = 2 1 )}\end{array}$ & $\begin{array}{l}\text { Total } \\
(\mathbf{n = 3 7 )}\end{array}$ & \multirow{2}{*}{ P-value } \\
\cline { 2 - 4 } & Value (\%) & Value (\%) & Value (\%) & \\
\hline Yes & $3(18.7)$ & $11(52.4)$ & $14(37.8)$ & \multirow{2}{*}{0.04} \\
No & $13(81.3)$ & $10(47.6)$ & $23(62.2)$ & \\
\hline
\end{tabular}

Table 3. Reinterventions.

\begin{tabular}{lllll}
\hline \multirow{2}{*}{ Reoperations } & $\begin{array}{l}\text { Tissucol use } \\
(\mathbf{n = 1 6 )}\end{array}$ & $\begin{array}{l}\text { No Tissucol use } \\
(\mathbf{n}=\mathbf{2 1})\end{array}$ & $\begin{array}{l}\text { Total } \\
(\mathbf{n = 3 7 )}\end{array}$ & P-value \\
\cline { 2 - 4 } & Value (\%) & Value (\%) & Value (\%) & \\
\hline Yes & $2(12.5)$ & $9(42.9)$ & $11(70.3)$ & 0.048 \\
No & $14(87.5)$ & $12(57.1)$ & $26(29.7)$ & \\
\hline
\end{tabular}

Table 4. Leaks and drainage.

\begin{tabular}{lllll}
\hline \multirow{2}{*}{ Leaks } & $\begin{array}{l}\text { Drain placement } \\
(\mathbf{n = 9 )}\end{array}$ & $\begin{array}{l}\text { No drain } \\
\text { placement }(\mathbf{n = 2 8})\end{array}$ & $\begin{array}{l}\text { Total } \\
(\mathbf{n = 3 7 )}\end{array}$ & \multirow{2}{*}{ P-value } \\
\cline { 2 - 4 } & Value (\%) & Value (\%) & Value (\%) & \\
\hline Yes & $3(33.3)$ & $11(39.3)$ & $14(37.8)$ & \multirow{2}{*}{0.54} \\
No & $6(66.7)$ & $17(60.7)$ & $23(62.2)$ & \\
\hline
\end{tabular}

Table 5. Mortality.

\begin{tabular}{lllll}
\hline \multirow{2}{*}{ Death } & $\begin{array}{l}\text { Tissucol use } \\
(\mathbf{n = 1 6 )}\end{array}$ & $\begin{array}{l}\text { No Tissucol use } \\
(\mathbf{n = 2 1 )}\end{array}$ & $\begin{array}{l}\text { Total } \\
(\mathbf{n = 3 7 )}\end{array}$ & \multirow{2}{*}{ P-value } \\
\cline { 2 - 4 } & Value (\%) & Value (\%) & Value (\%) & \\
\hline Yes & $1(6.3)$ & $4(19.0)$ & $5(13.5)$ & 0.27 \\
No & $15(93.8)$ & $17(81.0)$ & $32(86.5)$ & 0.27 \\
\hline
\end{tabular}

\section{Discussion}

The patients in the subgroup of rectal anastomosis in the clinical trial (PROTISSUCOL001) presented with a large number of leaks from the anastomosis. The sample size was 250 patients to achieve significant data, which was originally calculated for a rate of clinical and subclinical leaks higher than $10 \%$. However, in our study, we have achieved significant differences with 104 patients analysed and with 37 patients specifically for rectal anastomosis.

There are references in the literature that have made us think that fibrin-based biological adhesives could act to prevent anastomotic leakage [19-21]. It is clear, based on the high number of reports available [22,23], that these drugs prevent anastomosis leakage in experimental animals. It has also been demonstrated that they have no influence on the result of an anastomosis of the gastrointestinal tract with the pancreas [24,25].

Alternatively, there are a few studies that demonstrate or suggest that fibrin-based biological adhesives could have a positive influence on the healing of rectal anastomoses. Particularly, only the clinical trial conducted by Silecchia et al. on gastrojejunal anastomosis has shown a protective effect on gastrointestinal anastomoses $[19,20]$. Similarly, other clinical trials, without reaching significance, have suggested that biological adhesives may have a protective effect on risk anastomoses [21].

Based on all these literature references, our study aimed to show that fibrin-based biological adhesives have a protective effect on anastomotic dehiscence. Our clinical trial showed 
early that these adhesives reduce the risk of healing defects in rectal anastomosis in patients undergoing sigmoid or rectal cancer operations, with leak rates significantly different between the two study groups $(52.4 \%$ in the control group versus $18.7 \%$ in the study group, $\mathrm{p}=0.04)$.

The present study also demonstrates that the number of reoperations decreases significantly $(p=0.048)$ as a result of this reduction in healing defects, without having an influence on mortality. The long-term consequences caused by the excessive number of septic complications of anastomotic origin on the oncological outcome in the control group and their long-term influence on mortality will still need to be analysed.

The beginning and end of the clinical trial coincided with the learning curve of laparoscopy in the departments involved. This circumstance, which was linked to the increased diagnosis of the number of leaks by systematically conducting a control test with contrast, has led to a high rate of diagnoses of rectal anastomosis dehiscence. Although it is certainly a transient and undesirable situation, these learning curves have led to high patient morbidity. In our case, the increase in leakage rate during this period has allowed us to increase the number of adverse effects, leading to significant differences at an early stage. Otherwise, it would have been necessary to achieve the initially calculated sample size of 250 patients at a minimum.

Certain authors have postulated that these adhesives can act more as healing agents than as adhesive agents [26]. It is challenging to think that these substances, which are registered as drugs in their Summary of Product Characteristics, may lower the number of leaks in rectal anastomosis to less than half by a simple mechanical action. In this sense, it has never been demonstrated that the increased number of suture lines would reduce the risk of leakage [27]. However, there are reports already showing experimentally and clinically that the presence of growth factors or stem cells in certain media promotes the healing of certain tissues [28]. Therefore, we postulate, although it cannot be demonstrated by our study, that fibrin-based biological adhesives may act as a drug that induces tissue healing by reproducing the essential first step for the process to take place, i.e., the creation of a stable fibrin network by means of which migration and proliferation of fibroblasts is favoured by the presence of growth factors.

There is great controversy about the use or non-use of drains. In the overall results of our study, the use of drainage was an independent protective factor of the study groups for dehiscence. This protective factor was not demonstrated in only the subgroup of rectal anastomosis because, although the percentage trend might suggest it, the p-value indicates a high association with chance (Table 4). Conversely, it is important to remember that this was not a primary objective of the study and, therefore, was not a controlled factor. Adding to the controversy over these findings, recent reports point to the possibility that drains could favour anastomotic leakage [29,30]. Nevertheless, more controlled studies will be needed, whose primary objective would be to prove, or disprove this concept.
Fibrin-based biological adhesives are drugs that have been shown in our study to decrease the risk of leakage in rectal anastomosis to less than half. In addition, they have also decreased significantly the need for reoperation. These findings are sufficient to recommend their use systematically in rectal anastomosis. Further studies are needed to demonstrate the mechanism of action of these drugs by combining adhesives with a given concentration of growth factors or stem cells to further improve the results. If this trend continues through the end of the study, the use of these adhesives could not only reduce serious postoperative complications related to dehiscence in these patients but also improve the prognosis and oncological outcome of rectal and sigmoid cancer treatment.

\section{References}

[1] Schnüriger B, Inaba $\mathrm{K}, \mathrm{Wu} \mathrm{T}$, Eberle BM, Belzberg $\mathrm{H}$, Demetriades D. Crystalloids after primary colon resection and anastomosis at initial trauma laparotomy: excessive volumes are associated with anastomotic leakage. J Trauma. 2011;70:603-10.

[2] Poon RT, Fan ST. Decreasing the pancreatic leak rate after pancreaticoduodenectomy. Adv Surg. 2008;42:33-48.

[3] Ben-David K, Sarosi GA, Cendan JC, Howard D, Rossidis G, Hochwald SN. Decreasing morbidity and mortality in 100 consecutive minimally invasive esophagectomies. Surg Endosc. 2011;26:162-7.

[4] Ivanov D, Cvijanović R, Gvozdenović L. Intraoperative air testing of colorectal anastomoses. Srp Arh Celok Lek. 2011;139:333-8.

[5] Lin SC, Chen PC, Lee CT, et al. Routine defunctioning stoma after chemoradiation and total mesorectal excision: a single-surgeon experience. World J Gastroenterol. 2013; 19:1797-1804.

[6] Seo SI, Yu CS, Kim GS, et al. The role of diverting stoma after an ultra-low anterior resection for rectal cancer. Ann Coloproctol. 2013;29:66-71.

[7] Park JS, Choi GS, Kim SH, et al. Multicenter analysis of risk factors for anastomotic leakage after laparoscopic rectal cancer excision: the Korean laparoscopic colorectal surgery study group. Ann Surg. 2013;257:665-71.

[8] Smith JD, Butte JM, Weiser MR, et al. Anastomotic leak following low anterior resection in stage IV rectal cancer is associated with poor survival. Ann Surg Oncol. 2013;20:2641-6.

[9] Law WL, Choi HK, Lee YM, Ho JW, Seto CL. Anastomotic leakage is associated with poor long-term outcome in patients after curative colorectal resection for malignancy. J Gastrointest Surg. 2007;11:8-15.

[10] Arteaga-González IJ. A revolutionary design change to improve stapler safety. Obes Surg. 2013;23:112-4.

[11] Honda M, Kuriyama A, Noma H, Nunobe S, Furukawa TA. Hand-sewn versus mechanical esophagogastric anastomosis after esophagectomy: a systematic review and meta-analysis. Ann Surg. 2013;257:238-248. 
[12] Sajid MS, Hutson KH, Rapisarda IF, Bonomi R. Fibrin glue instillation under skin flaps to prevent seroma-related morbidity following breast and axillary surgery. Cochrane Database Syst Rev. 2013;5:CD009557.

[13] Kobayashi S, Nagano H, Marubashi S, et al. Fibrin sealant with PGA felt for prevention of bile leakage after liver resection. Hepatogastroenterology. 2012;59:2564-8.

[14] Sanders DL, Waydia S. A systematic review of randomised control trials assessing mesh fixation in open inguinal hernia repair. Hernia. 2013 May 7. [Epub ahead of print].

[15] Tolver MA, Rosenberg J, Juul P, Bisgaard T. Randomised clinical trial of fibrin glue versus tacked fixation in laparoscopic groin hernia repair. Surg Endosc. 2013;27:2727-33.

[16] Prieto-Díaz-Chávez E, Medina-Chávez JL, Ramírez-Barba EJ, Trujillo-Hernández B, Millán-Guerrero RO, Vásquez C. Reduction of peritoneal adhesion to polypropylene mesh with the application of fibrin glue. Acta Chir Belg. 2008;108:433-7.

[17] Giuratrabocchetta S, Rinaldi M, Cuccia F, et al. Protection of intestinal anastomosis with biological glues: an experimental randomised controlled trial. Tech Coloproctol. 2011;15:153-8.

[18] Herreros MD, Garcia-Arranz M, Guadalajara H, De-La-Quintana P, Garcia-Olmo D. FATT Collaborative Group: Autologous expanded adipose-derived stem cells for the treatment of complex cryptoglandular perianal fistulas: a phase III randomised clinical trial (FATT 1: fistula Advanced Therapy Trial 1) and long-term evaluation. Dis Colon Rectum. 2012;55:762-72.

[19] Silecchia G, Boru CE, Mouiel J, et al. Clinical evaluation of fibrin glue in the prevention of anastomotic leak and internal hernia after laparoscopic gastric bypass: preliminary results of a prospective, randomised multicenter trial. Obes Surg. 2006;16:125-131.

[20] Silecchia G, Boru CE, Mouiel J, et al. The use of fibrin sealant to prevent major complications following laparoscopic gastric bypass: results of a multicenter, randomised trial. Surg Endosc. 2008;22:2492-7.
[21] Fernandez Fernandez L, Tejero E, Tieso A. Randomised trial of fibrin glue to seal mechanical oesophagojejunal anastomosis. Br J Surg. 1996;83:40-1.

[22] De Almeida JR, Ghotme K, Leong I, Drake J, James AL, Witterick IJ. A new porcine skull base model: fibrin glue improves strength of cerebrospinal fluid leak repairs. Otolaryngol Head Neck Surg. 2009;141:184-9.

[23] Bonanomi G, Prince JM, McSteen F, Schauer PR, Hamad GG. Sealing effect of fibrin glue on the healing of gastrointestinal anastomoses: implications for the endoscopic treatment of leaks. Surg Endosc. 2004;18:1620-4.

[24] Suc B, Msika S, Fingerhut A, et al. Temporary fibrin glue occlusion of the main pancreatic duct in the prevention of intra-abdominal complications after pancreatic resection: prospective randomised trial. Ann Surg. 2003;237:57-65.

[25] Lillemoe KD, Cameron JL, Kim MP, et al. Does fibrin glue sealant decrease the rate of pancreatic fistula after pancreaticoduodenectomy? Results of a prospective randomised trial. J Gastrointest Surg. 2004;8:766-74.

[26] Morikawa T. Tissue sealing. Am J Surg. 2001;182:29S-35S.

[27] Baker RS, Foote J, Kemmeter P, Brady R, Vroegop T, Serveld M. The science of stapling and leaks. Obes Surg. 2004;14:1290-8.

[28] Herreros MD, Garcia-Arranz M, Guadalajara H, De-La-Quintana P, Garcia-Olmo D; FATT Collaborative Group. Autologous expanded adipose-derived stem cells for the treatment of complex cryptoglandular perianal fistulas: a phase III randomised clinical trial (FATT 1: fistula Advanced Therapy Trial 1) and long-term evaluation. Dis Colon Rectum. 2012;55:762-72.

[29] Salgado W Jr, Cunha Fde Q, dos Santos JS, et al. Routine abdominal drains after Roux-en-Y gastric bypass: a prospective evaluation of the inflammatory response. Surg Obes Relat Dis. 2010;6:648-52.

[30] Akiyoshi T, Ueno M, Fukunaga Y, et al. Incidence of and risk factors for anastomotic leakage after laparoscopic anterior resection with intracorporeal rectal transection and double-stapling technique anastomosis for rectal cancer. Am J Surg. 2011;202:259-64. 